\title{
Recurent Ectopic Pregnancy Score As Predictor To Fertility Outcome Following Tubal Ectopic Pregnancy
}

\author{
Nurul Yaqin Mohd Nor ${ }^{1,2}$ \\ ${ }^{1}$ Department of Obstetric and Gynaecology, Kulliyyah of Medicine, International \\ Islamic University Malaysia \\ ${ }^{2}$ Department of Obstetric and Gynaecology, Hospital Sultanah Nur Zahirah Kuala \\ Terengganu
}

Presenter: Nurul Yaqin Mohd Nor

Introduction: Ectopic pregnancy may occur in fallopian tube, ovary or abdominal cavity. This study focused on tubal pregnancy outcome. The return of fertility after operative removal of tubal ectopic pregnancy was examined in 237 women who were admitted to Hospital Sultanah Nur Zahirah (HSNZ) for ectopic pregnancy between 2012 and 2016. The follow-up period averaged six months to four years. Materials and Methods: This was a case control, retrospective non-comparative study. Data collected from HSNZ Human Resources Management Information System (HRMIS). Patient who has no pregnancy or delivery record after six months of ectopic event were contacted via phone. Multivariate analysis was done to identify score for patients who were at risk of recurrence, and who require fertility help. Results: One hundred forty eight women (64\%) aged 20-35 years old able to conceive spontaneously after ectopic pregnancy, with the mean-time of 8 months if one tube is patent. Ninety three percents of those who conceived had intrauterine pregnancy, $5 \%$ had recurrent ectopic pregnancy. Conclusion: From a multivariate analysis, a scoring system, Recurrent Ectopic Pregnancy (REP) score was made to choose most suitable patient for laparoscopic intervention, preserve fertility or facilitate fertility. Significant REP score of 4/5 suggest laparoscopic salpingectomy with contralateral sterilization to prevent recurrence of ectopic. Patient with REP score 2-3 may have spontaneous conception and REP score less than $1 / 5$ will require further fertility treatment. 